\title{
Numerical Indicators of Absorption Spectra of Leaf Extracts Obtained from Conifer Family Plants
}

\author{
Vladimir Mikhaylovitch Koldaev", Marina Sergeevna Titova \\ Federal Scientific Centre of the East Asia Terrestrial Biodiversity, Vladivostok, Russia \\ Email address: \\ kolvm42@rambler.ru (V. M. Koldaev) \\ ${ }^{*}$ Corresponding author \\ To cite this article: \\ Vladimir Mikhaylovitch Koldaev, Marina Sergeevna Titova. Numerical Indicators of Absorption Spectra of Leaf Extracts Obtained from \\ Conifer Family Plants. American Journal of Agriculture and Forestry. Vol. 7, No. 3, 2019, pp. 106-110. doi: 10.11648/j.ajaf.20190703.13
}

Received: March 21, 2019; Accepted: April 27, 2019; Published: June 12, 2019

\begin{abstract}
The purpose of this study is to examine the ratios of absorption by photosynthetic complex substances and other components of leaf extracts obtained from conifer family plants of different genera. Such ratios were determined by absorption spectrometry method using unconventional numerical values of absorption spectra, such as wave length of the highest maximum, coefficient of relative photoabsorption, blue-red coefficient, total absorption intensity and relative share of absorption by photosynthetic complex substances in total absorption. The intensity of light energy's utilization by conifers was shown to be the highest among conifers of pine family, to be the lowest among spruce family plants and to have intermediate values among plants of fir and cypress genera. Numerical indicators of absorption spectra used in this article reflect the properties of photoreceptive system of coniferous plants and could be used in photosynthesis studies and also as criteria in environmental science and plant resources monitoring during assessment of the impact anthropogenic, climate and other factors have on coniferous forests.
\end{abstract}

Keywords: Photosynthesis, Spectrophotometry, Spruce, Fir, Pine, Chamaecyparis Lawsoniana

\section{Introduction}

Photosynthesis mechanisms [1,2] and properties of basic pigments of the photosynthetic complex (PSC) of chlorophyll and carotenoids, which absorb in a visible section of the optical spectrum, have so far been thoroughly studied [3, 4]. Comparatively less attention is paid to other leaf components (OLC) not included into the photosynthetic system, such as anthocyans [5], coumarins and saponins, which absorb ultraviolet light. The ratios of absorption by PSC substances and OLC are determined by absorption spectrophotometry methods using numerical indicators of absorption spectra (NIAS) $[6,7]$ and could be used as photoabsorption characteristics [8]. However, in case of conifer family plants such rations are poorly studied and underrepresented in scientific literature.

Currently, it is important to use NIAS to compare the intensity of light absorption by the leaves of coniferous trees, which prefer different habitats in the forest. The purpose of this study is to examine the rations of absorption by PSC substances and other components of leaf extracts obtained from conifer family plants of different genera. To achieve the goal, it is advisable to use coniferous trees, preferring growth conditions with different illumination. Spruce trees usually prefer shaded areas, pines grow in lighter areas, silver fir and cypress trees grow in areas with medium light. Research can be performed on the following plan: 1) select several species of spruce, fir, pine and cypress for comparison, 2) to conduct spectrophotometry of extracts from the leaves of these trees, 3 ) to determine the numerical indicators of the absorption spectra of the extracts, 4) to compare the obtained numerical indicators and 5) to conclude on the priorities of absorption activity among conifers.

\section{Material and Methods}

10 species of 25-30-year-old coniferous plants belonging to 4 genera and growing in the environment specific to the southern part of Primorsky Krai, Russia, were used for this study. In July 4 to 6 independent samples of new needles were taken from each plant species in the afternoon under dry weather conditions, followed by immediate production of 
their extracts in a shaded room. Needle bunches were grinded in a porcelain jar filled with quartz sand, magnesium carbonate and $95 \%$ ethanol. In 5 to 6 minutes the extracts were filtered through PTGE-H $0.45 \mu \mathrm{m}$ filter (HyundaiMicro, Republic of Korea) in to dark glass flask. A digital spectrophotometer UV-2501PC (Shimadzu, Japan) was used within range of 220 to $700 \mathrm{~nm}$ with a $1 \mathrm{~nm}$ interval to register absorption spectra. The spectra were rated by the highest maximum. The results were statistically treated using small sample methods [9].

The first-order derivative of the normalized absorption spectrum (NAS) was calculated using numerical method. The coordinates of inflection points of the absorption band contour were determined by an absolute value of local maximums (Figure 1, points 1, 2 and 3). NIAS used in the study are as follows:

Wave length $\lambda_{\mathrm{HM}}$ of the highest maximum $(H M)$;

Coefficient of relative photoabsorption $(C R P)$, equal to the ratio of extinction $A_{R}$ at the analytical wave length of chlorophyll absorption in the red section to extinction $A_{H M}$ at the wave length of the highest maximum (Figure 1):

$$
C R P=\frac{A_{R}}{A_{H M}}
$$

blue-red coefficient $(B R C)$ was calculated as the ratio of extinction $A_{B}$ at the blue wave length and extinction $A_{R}$ at the red one of chlorophyll absorption:

$$
B R C=\frac{A_{B}}{A_{R}}
$$

The integral absorption intensity of the OLC corresponds to the area $S_{\mathrm{OLC}}$, limited by the band contour in the ultraviolet section. The area was calculated in accordance with Simpson's rule [10] within range between $\lambda_{1}$ (inflection point1) near the maximum of $A_{\mathrm{HM}}$ and $\lambda_{2}$ (inflection point2) on the left slope of $A_{\mathrm{B}}$ 's "blue" maximum contour (Figure 1, diagonal hatching). The integral intensity of absorption by PSC substances, which was numerically equal to the area $S_{\text {PSC }}$ under the contour of absorption band's visible range (Figure 1, vertical hatching), was calculated in the same way, using integration range between $\lambda_{2}$ (inflection point 2) on the left slope of $A_{\mathrm{B}}$ 's contour and $\lambda_{3}$ (inflection point 3 ) near the $A_{\mathrm{R}}$ 's "red" maximum. The total intensity of absorption $\left(S_{\mathrm{T}}\right)$ of a whole leaf was taken as a sum of the foregoing areas:

$$
S_{\mathrm{T}}=S_{\mathrm{OLC}}+S_{\mathrm{PSC}}
$$

The relative share $(F)$ of absorption by PSC substances in total absorption by a leaf was expressed in percentage points:

$$
F=100 \frac{S_{\mathrm{PSC}}}{S_{\mathrm{T}}}(\%)
$$

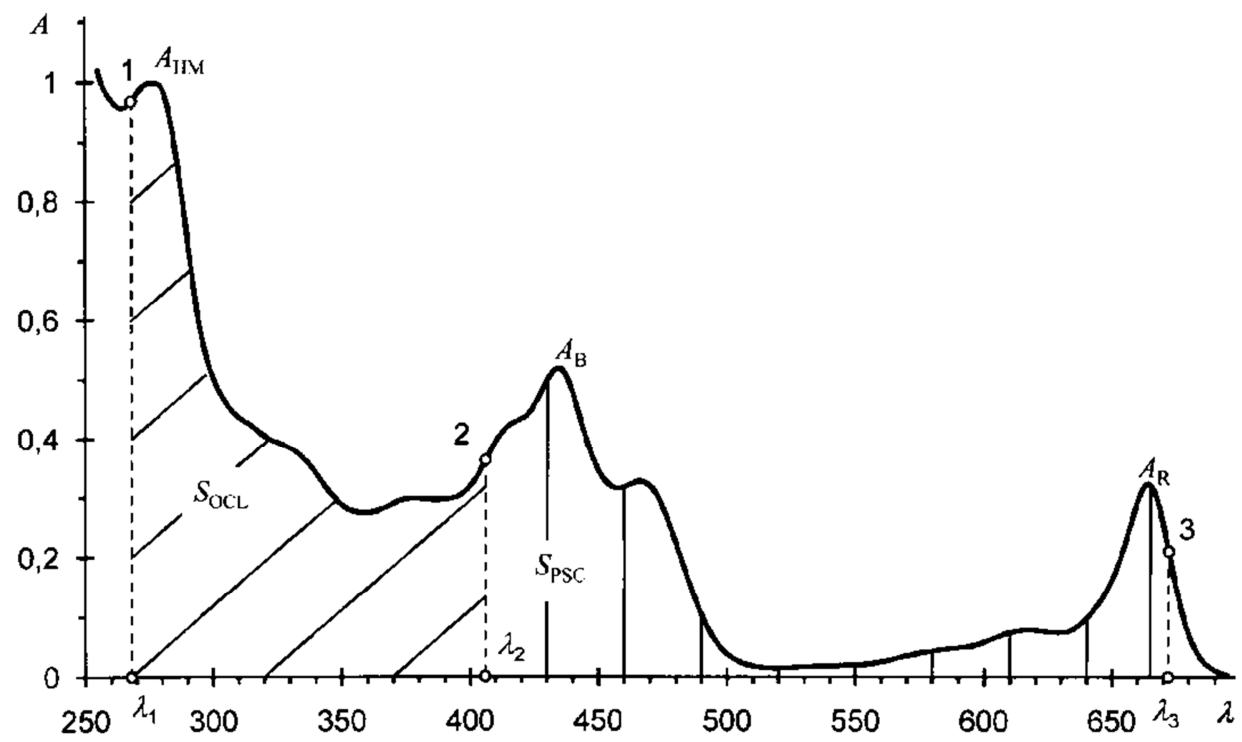

Figure 1. Normalized absorption spectrum of Picea ajanensis (Fisch. ExCarr.) extract. 1, 2 and 3 are inflection points of the band contour. $A_{H M}$, $A_{B}$ and $A_{R}$ are extinctions of the highest, blue and red maximums; $\lambda_{1}, \lambda_{2}$ an $d \lambda_{3}$ are $x$-coordinates inflection points; $S_{O L C}$ and $S_{P S C}$ areas corresponding to intensity of absorption by OLC and PSC substances. X-axis: wavelength $\lambda$ in $n m ; y$-axis-extinction A in relative units.

All calculations were carried out using original software $[11,12]$.

\section{Results}

Absorption spectra of the same type were obtained for needle extracts produced from all studied plants (Figure 2). The visible range of registered NAS included four maximums at the wave length of $434 \pm 1 ; 466 \pm 2 ; 618 \pm 1$ and $664 \mathrm{~nm}$. At these wave length values the lowest extinctions were obtained for spruce needles, medium ones for fir and cypress needles, and the highest ones for pine needles.

As the study results show (see Table 1), $\lambda_{\mathrm{HM}}$ in the needle extracts' NAS is within ultraviolet range between 272 and $282 \mathrm{~nm}$. The lowest $\lambda_{\mathrm{HM}}$ values were reported for spruces, with their mean value of $272 \pm 1.04 \mathrm{~nm}$. The mean $\lambda_{\mathrm{HM}}$ values in the fir and pine extracts' NAS exceed that of spruces by 3 to $5 \mathrm{~nm}$, and the mean $\lambda_{\mathrm{HM}}$ value in Chamaecyparis lawsonianaextract's NAS exceeds it by $10 \mathrm{~nm}$.

The lowest CRP (formula (1)) values averaging 
$0.084 \pm 0.005$ were obtained for spruce needle extracts' NAS. The mean CRP values of the fir and cypress extracts and the pine needle extracts exceed those of the spruce needle extracts 1.54 to 1.61 times and 2.92 times respectively.
The lowest $S_{\mathrm{T}}$ (formula (3)) values were reported for spruce needle extracts averaging $65.57 \pm 2.15$, and similar mean values were also reported for the fir and pine extracts, i.e. $64.25 \pm 1.64$ and $67.87 \pm 2.78$ respectively.

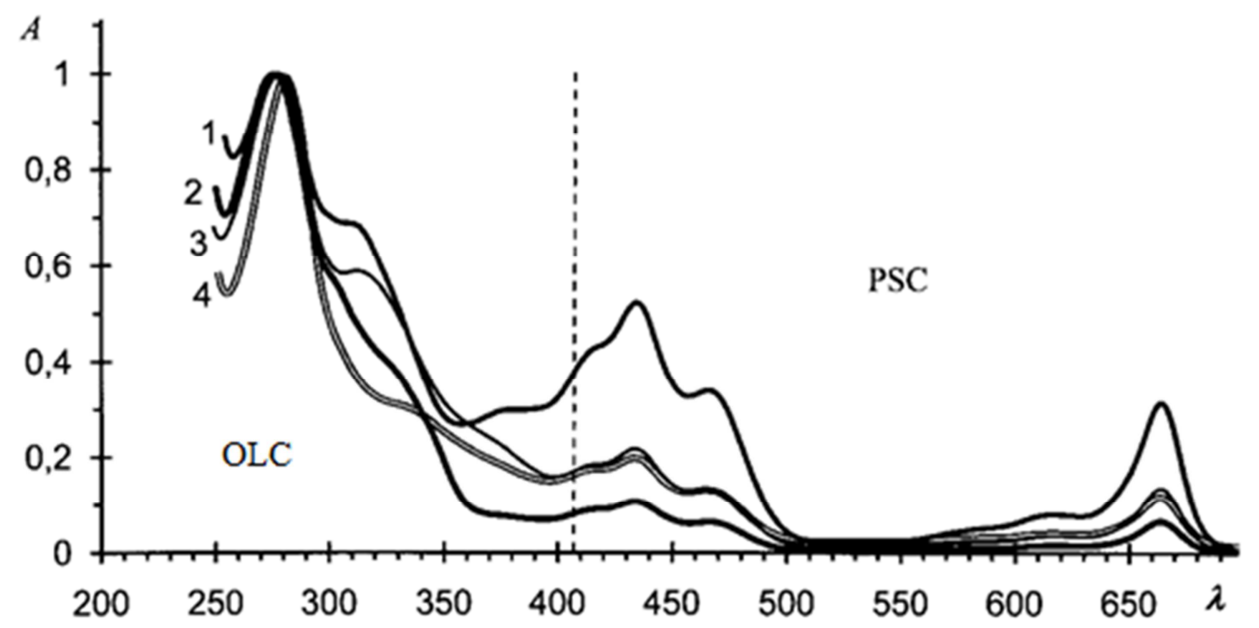

Figure 2. Examples of normalized absorption spectra of needle extracts obtained from Pinus koraiensis (1), Picea smithiana (2), Abies koreana (3) and Chamaecyparis lawsoniana (4). PSC: area of absorption by photosynthetic complex substances, OLC: area of absorption by other leaf components. X-axis: wave length $\lambda$ in $\mathrm{nm} ; \mathrm{y}$-axis-extinction $\mathrm{A}$ in relative units.

Table 1. Wave length of the highest maximum $\left(\lambda_{H M}\right)$, coefficient of relative photoabsorption $(C R P)$, blue-red coefficient $(B R C)$, total intensity of absorption $\left(S_{T}\right)$ and share $(F)$ of absorption by photosynthetic complex substances in total absorption for absorption spectra of needle extracts obtained from trees of different species.

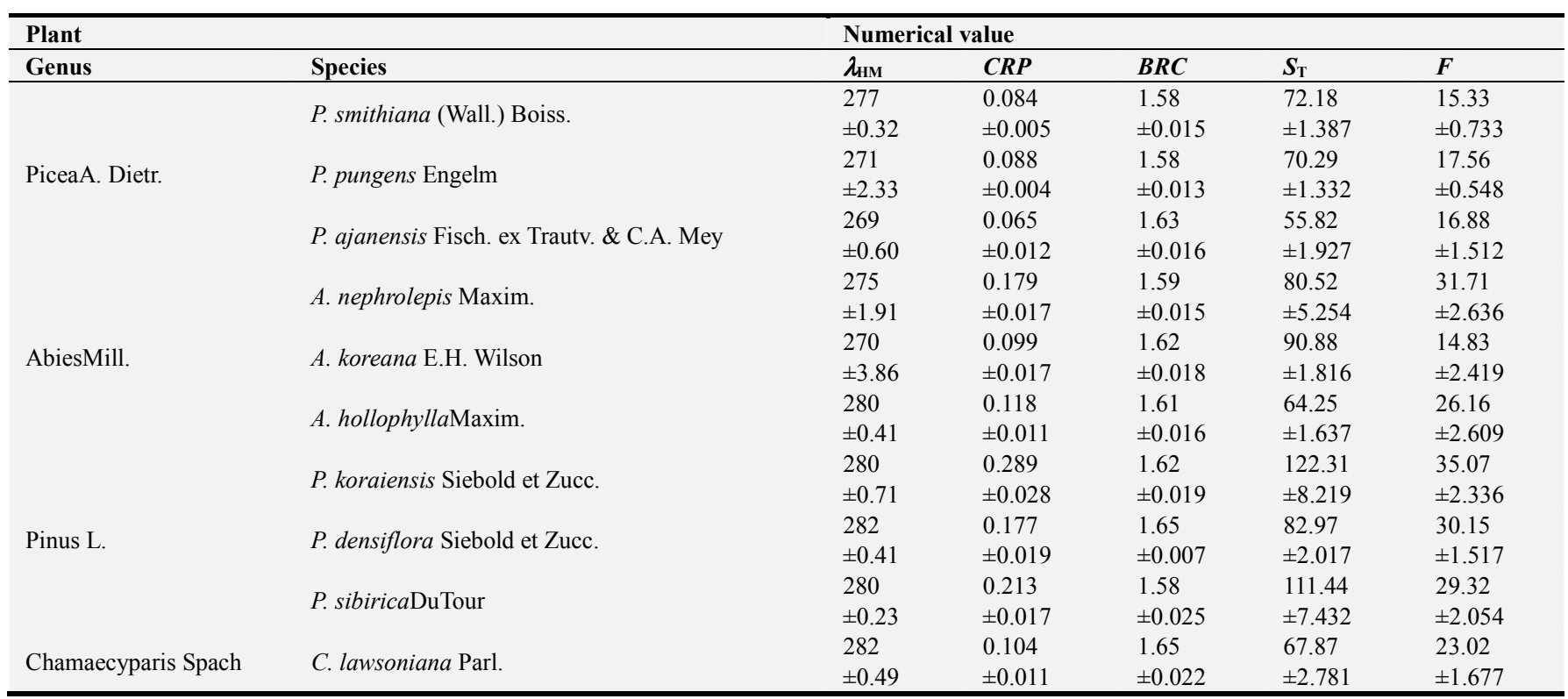

Each value is expressed as mean \pm square error of mean $(\mathrm{n}=6)$

The mean $S_{\mathrm{T}}$ value of the pine needle extracts exceeded that of the spruce needle ones 1.6 times $(p<0,001)$. The share of absorption by photosynthetic complex substances in total absorption for the spruce needle extracts averaged $(16.47 \pm 0.65) \%$. The mean $F$ value for the fir and cypress needle extracts and the pine needle extracts exceeded that for the fir needle ones 1.40 to 1.46 times and 1.93 times respectively.

The obtained results show that $B R C$ (formula (2)) values for the needle extracts obtained from all studied plants are within range of 1.58 to 1.64 , with the mean value of $1.61 \pm 0.06$, while the $B R C$ variation reached $0.37 \%$ and the confidence interval boundaries were 1.598 and 1.622 .

According to the obtained data, the highest mean NIAS values of the extracts obtained from pine genus trees, such $F$ (formula (4)) and $C R P$, exceed those obtained from spruce genus ones by 48.2 to $65.8 \%$, while in case of such NIAS as $B R C$ and $\lambda_{\mathrm{HM}}$ the excess is only 1.2 and $3.5 \%$ respectively.

\section{Discussion}

As the study results show, the shapes of absorption band 
contours of NAS of theneedle extracts obtained from different coniferous tree species are quite similar to each other and the corresponding maximums are similar to each other in terms of wave lengths (Figure 2), while their set matches the maximums of chlorophyll absorption [13]. Such similarity is due to the qualitative identity of PSC substances in leaves of different plant species [1]. Moreover, $B R C$ numerical value is virtually the same for all studied coniferous plant species, which is also confirmed by the constancy of PSC substances in their needles.

In all studied needle extracts' NAS the $\lambda_{\mathrm{HM}}$ value is within ultraviolet range, which is consistent with our previously obtained data $[6,8]$ for the leaf extracts of plants and broadleaved trees. The fact that the highest maximums are within ultraviolet range is apparently a feature of absorption spectra of leaf extracts of all terrestrial green plants and is probably due to the optical properties of flavonoids, tannins, saponins etc. of the OLC, which absorb exactly in this range. Yet, in case of conifers the contribution to absorption in the extracts' NAS is probably also made by resins present in the needles, which content in, say, pine needles may be as high as 7 to $12 \%$.

The numerical $C R P$ value by default depends on the ration of extinctions at the red wave and $\lambda_{\mathrm{HM}}$, yet since the extinction $A_{\mathrm{HM}}$ in the NAS is unity, the $C R P$ value reflects a relevant absorption by chlorophylls, while higher $C R P$ values point to increased photoreception of their needle extract and lower ones, to the contrary, point to lower photoreception. According to the obtained data, the highest and the lowest $C R P$ values are specific to pines and spruces respectively, while other studied trees belong to an intermediate position in terms of this indicator.

The total intensity of absorption $S_{\mathrm{T}}$ provides an image of cumulative absorption of all leaf components within examined range. The most interesting part is undoubtedly the area of absorption carried out by photosynthetic complex substances, and this part is reflected by the $F$ value expressed in percentage points as the ratio of intensities of absorption by PSC substances and total one. As the obtained data show, pines are featured by higher $S_{\mathrm{T}}$ and $F$ values respectively and also stronger ability to absorb light energy by PSC substances, while spruces are featured by the lowest ones and other studied trees belong to an intermediate position in terms of these indicators.

Thus, in terms of aggregated NIAS increase and, accordingly, in ascending order of the light utilization level of their needle extracts the studied tress could be lined up as follows:

[Picea spruces] < Abies firs and Chamaecyparis cypress $]<$ [Pinus pines].

Since the NIAS used in this study have not been previously used for the spectrophotometric analysis of needle extracts, direct comparisons with literature data are excluded. However, there may be correlations with published data on biochemistry and physiology of coniferous plants. For example, a number of studies show lower levels of photochemical processes in the needles of different spruce species [14-18] comparing with firs [19, 20] and pines [21] under normal conditions. At the same time, it was found out that pine needles [22] were featured by higher photosynthesis intensity that that of cypress needles [23, 24]. In other words, pines and spruces are featured by higher and the lowest levels of photosynthetic processes respectively, while other studied coniferous plants belong to an intermediate position in terms of photosynthetic process level, which is quite consistent with the presented results, which were obtained in this study.

The used NIAS reflect the properties of plants' photoreceptive system. The most valid ones among the used NIAS are $F$ and $C R P$ values, which are appropriate to be used in further studies.

It should be noted that, comparing to, say, liquid chromatography [25], the used method of absorption spectrophotometry is less time-consuming and featured by quite high accuracy and also does not require additional reagents (bar extraction agents), which is quite important for the field practice of plant studies.

\section{Conclusions}

Using unconventional numerical indicators for the analysis of light absorption by plant leaves extends the field of applications of molecular spectrophotometry, which is important for increasing the amount of useful data extracted with a spectrophotometer and for further development of methodology of absorption spectrophotometry.

The experimental material presented in this study demonstrates a new approach to a spectrophotometric assessment of light absorption by plant leaves, which could be dubbed "method of numerical indicators of absorption spectra" or, in short, "NIAS method". The results obtained using the NIAS method is not at odds with modern perceptions about biochemistry of coniferous plants.

The obtained results point to the prospects of using unconventional numerical indicators, especially $C R P$ and $F$ ones, as criteria in plant resources monitoring during assessment of the impact anthropogenic, climate and other factors have on coniferous trees.

\section{Confirmation}

The authors would like to express their gratitude to unknown readers for their review and useful comments.

\section{Financing}

The authors hereby report that had no any sponsorship or financing other than government subsidies when carrying out this study.

\section{Contribution of Authors}

All the authors have equally contributed to this study. 


\section{Ethics}

This is an original article that contains unpublished materials. The authors hereby confirm that there are no any ethical issues.

\section{References}

[1] Blankenship R. E. Molecular mechanisms of photosynthesis / John Wiley \& Sons: Washington, 2014.-312 p.

[2] Niyogi K. K. Safety valves for photosynthesis // Current Opinion in Plant Biology (2000), 3 (6), 455-460.

[3] Mishra S. R. Photosynthesis in Plants /Discovery Publishing House: New Delhi, 2004.-296 p.

[4] Holik L., Niineme Ü., Kull O. Photosynthetic acclimation to light in woody and herbaceous species: a comparison of leaf structure, pigment content and chlorophyll fluorescence characteristics measured in the field // Plant biology (2012), 14 (1), 88-99.

[5] Oren-Shamir M. Does anthocyanin degradation play a significant role in determining pigment concentration in plants? // Plant Science (2009), 177 (4), 310-316.

[6] Koldaev V. M. The variety of forms of absorption spectrums of ethanol extracts from green leaves //Proceedings of the Samara Scientific Center of the Russian Academy of Sciences (2014), 16 (5/3), 1793-1795.

[7] Koldaev V. M., Manyakin A. Y. Numerical indicators of absorption spectra of green leaf extract obtained from plants of different forms // Spectrochemica Acta Part A: Molecular and Biomolecular Spectroscopy (2018), 203, 404-407.

[8] Koldaev V. M. The seasonal change of relative photoabsorption in green leaves of plants // Proceedings of the Samara Scientific Center of the Russian Academy of Sciences (2016), 18 (2), 101-104.

[9] McDonald, J. H. Handbook of biological statistics / Sparky House Publishing: Baltimore, 2014.-326 p.

[10] Composite accessed: 12.03 .2019

Simpson's Date

[11] Koldaev V. M., Zorikov P. S., Bezdetko G. N. Spectra. Electronic bulletin of programs for computers, databases, $\begin{array}{llll}\text { circuit layouts, } & 2009, & \text { No } & 4\end{array}$ http://www.fips.ru/Electronic_bulletin/Programs_db_topology /01_PR/pdfDate accessed: 06.03.2019.

[12] Koldaev V. M., Zorikov P. S., Titova M. S. Simpson-2. Electronic bulletin of programs for computers, databases, $\begin{array}{llll}\text { circuit layouts, 2017, No } & \text { N. }\end{array}$ http://www1.fips.ru/wps/PA_FipsPub/res/BULLETIN/PrEVM /2017/05/20/INDEX.HTMDate accessed: 05.01.2019.

[13] Seely G. R., Jensen R. G. Effect of solvent on the spectrum of chlorophyll // Spectrochimica Acta (1965), 21, 1835-1845.

[14] Benomar L., Lamhamedi M. S., Villeneuve I., Rainville A., Beaulieu J., Bousquet J., Margolis H. S. Fine-scale geographic variation in photosynthetic-related traits of Picea glauca seedlings indicates local adaptation to climate // Tree Physiology (2015), 35 (8), 864-878.

[15] Manuel G. G., Colom R., Minotta G. Effects of nutrient supply on photosynthetic acclimation and photo inhibition of one-year-old foliage of Picea abies // Physiologia Plantarum (2001), 111 (2), 245-254.

[16] Stinziano J. R., Hüner N. P. A., Way D. A. Warming delays autumn declines in photosynthetic capacity in a boreal conifer, Norway spruce (Picea abies) // Tree Physiology (2015), 35 (12), 1303-1313.

[17] Walcroft A. S., Whitehead D., Kelliher F. M., Arneth A., Silvester W. B. The effects of long-term, partial shading on growth and photosynthesis in Pinus radiata D. Don trees // Forest Ecology and Management (2002), 163 (1-3), 151-163.

[18] Weng J-H., Liao T-S., Sun K-H., Chung J-C., Lin C-P., Chu C-H. Seasonal variations in photosynthesis of Picea morrisonicola growing in the subalpine region of subtropical Taiwan // Tree Physiology (2005), 25 (8), 973-979.

[19] Grassi G., Bagnaresi U. Foliar morphological and physiological plasticity in Picea abies and Abies alba saplings along a natural light gradient // Tree Physiology (2001), 21 (12-13), 959-967.

[20] Robakowski P., Wyka T., Samardakiewicz S., Kierzkowski D. Growth, photosynthesis, and needle structure of silver fir (Abies alba Mill.) seedlings under different canopies // Forest Ecology and Management (2004), 201 (2-3), 211-227.

[21] Niinemets Ü. Stomatal conductance alone does not explain the decline in foliar photosynthetic rates with increasing tree age and size in Picea abies and Pinus sylvestris // Tree Physiology (2002), 22 (8), 515-535.

[22] Warren C. R., Adams M. A. Distribution of N, Rubisco and photosynthesis in Pinus pinaster and acclimation to light // Plant, Cell and Environment (2001), 24 (6), 597-609.

[23] Han Q., Chiba Y. Leaf photosynthetic responses and related nitrogen changes associated with crown recosure after thinning in a young Chamaecyparis obtusa stand // Journal of Forest Research (2009), 14, 349-357.

[24] Kobayashi H., Inoue S., Gyokusen K. Photosynthesis-nitrogen relationship in a Hinoki cypress (Chamaecyparis obtusa) canopy: a comparison with Japanese cedar (Cryptomeria japonica) // Photosynthetica (2012), 50 (2), 317-320.

[25] AlKhalidi B. A., Shtaiwi M., AlKhatib H. S., Mohammad M., Bustanji Y. A comparative study of first-derivative spectrophotometry and column high-performance liquid chromatography applied to the determination of repaginate in tables and for dissolution testing. // Journal of AOAC International (2008), 91 (3), 530-535. 